\title{
Kondo physics of magnetic adatoms on metallic surfaces when the onset of the surface conduction density of states crosses the Fermi level
}

\author{
J. Fernández 1 and P. Roura-Bas \\ Centro Atómico Bariloche, CNEA, 8400 S. C. de Bariloche, Argentina
}

(Received 16 August 2019; revised manuscript received 3 October 2019; published 23 October 2019)

\begin{abstract}
We study the role of the onset of Shockley states $D_{s}$ belonging to (111) surfaces of $\mathrm{Cu}, \mathrm{Ag}$, and $\mathrm{Au}$ in the Kondo effect when a magnetic impurity is deposited on them. When $D_{s}$ approaches the Fermi level $E_{F}$, which can be done by compressing (stretching) the metallic sample, we find that most of the thermodynamic and dynamic properties of the impurity are affected in a nontrivial way. We model the system by a generic Anderson impurity model and solve it by using the numerical renormalization group technique. In particular, the impurity contribution to magnetic susceptibility and entropy as a function of temperature exhibit negative values and go to zero slowly in a logarithmic shape. Furthermore, we found suppression of the spectral density weight at the Fermi level when $D_{s} \sim E_{F}$ even in the Kondo regime. As a consequence, the conductance through the impurity is strongly reduced by nearly $25 \%$ of the unitary value $2 e^{2} / h$. Finally, we analyze these features in realistic systems like $\mathrm{Co}$ on $\operatorname{Ag}(111)$ reported in the literature.
\end{abstract}

DOI: 10.1103/PhysRevB.100.165139

\section{INTRODUCTION}

In solid-state physics, Shockley states arise when solving the Schrödinger equation in the context of nearly-free-electron models as a consequence of crystal termination. They are a common feature of (111) surfaces of noble metals [1]. In the case of $\mathrm{Ag}, \mathrm{Cu}$, and $\mathrm{Au}$ metals, the $d$-surface bands are well below the Fermi energy $E_{F}$, while the $s p$-surface ones are located around $E_{F}[2]$.

The nearly constant surface density of states (SDOS) abruptly starts below $E_{F}$ at $D_{s}-E_{F} \approx-450 \mathrm{meV}$ for $\mathrm{Cu}$ [3], $D_{s}-E_{F} \approx-475 \mathrm{meV}$ for Au [4], and $D_{s}-E_{F} \approx-67 \mathrm{meV}$ for $\mathrm{Ag}$ [5]. From scanning tunneling microscope (STM) measurements, the corresponding steps have been observed [4-6]. Interestingly, the occupation of these surface states depends appreciably on particular variables such as temperature and the presence of adsorbed species and, more importantly, on stretching the sample. Decreasing temperature, the onset of $\operatorname{Ag}(111)$ SDOS moves monotonically towards $E_{F}$ [7]. Stretching the Ag layers, induced by the film growth, shifts $D_{s}$ up in energy, even beyond the Fermi level [8]. Furthermore, the onset of SDOS can be changed by alloying the different noble metals at the surface $[4,9]$. In addition, the construction of a piezoelectric-based apparatus for applying continuously tunable compressive and tensile strains to test samples is reported in Ref. [10]. It can be used within a wide temperature range, including cryogenic ones $[10,11]$. In particular, such a device can be used for moving $D_{s}$ continuously across $E_{F}$.

On the other hand, the above-mentioned metal surfaces are often used to host magnetic impurities (see Fig. 1). Several experiments were made to study atomic impurities (such as Co or Mn) over such surfaces [3,5,12-18]. Moreover, complex magnetic molecules were also deposited on $\mathrm{Ag}, \mathrm{Cu}$, and $\mathrm{Au}$ surfaces like FePc [19,20], among others. With the help of low-temperature STM measurements, in these systems the
Kondo effect has been studied. In such measurements, the Kondo phenomenon emerges trough a narrow Fano-Kondo antiresonance in the differential conductance $G(V)=d I / d V$, where $I$ is the current and $V$ is the applied voltage.

The width of $G(V)$ is usually related to the Kondo temperature $T_{K}$, and below it the magnetic moment of the impurity is screened by the conduction electrons [21].

The Kondo effect has the quality of universality in the sense that most of the impurity properties display a universal shape once they are scaled by $T_{K}$. This fact makes $T_{K}$ the only relevant energy scale of the problem, and its precise estimation is always desirable.

Having mentioned that the onset of (111) surfaces can be move towards the Fermi energy and taking into account that the Kondo effect is a low-energy phenomenon, the question of how it is affected when $\left|D_{s}-E_{F}\right| \sim T_{K}$ is raised. Interestingly, when $\left|D_{s}-E_{F}\right| \gg T_{K}$, the Kondo temperature as a function of $D_{s}$ was found to display a power law, $T_{K} \simeq C\left|D_{s}-E_{F}\right|^{\alpha}[22]$.

In this paper we analyze the Kondo physics of a magnetic impurity on metallic surfaces when the onset of the surface conduction density of states crosses the Fermi level. We found that $T_{K}$ loses the power law dependence and, more importantly, both thermodynamic and transport properties of the impurity in the Kondo regime are strongly modified in a nontrivial way.

Modeling the system with an Anderson impurity Hamiltonian, we employ the numerical renormalization group (NRG) technique as implemented in the NRG LJUBLJANA open-source code [23].

In particular, when $\left|D_{s}-E_{F}\right| \leqslant T_{K}$, we found unusual temperature dependencies in the impurity properties like negative values of entropy $S_{\text {imp }}(T)$ and magnetic susceptibility $\chi_{\text {imp }}(T)$. Furthermore, since $D_{s}$ can be moved towards $E_{F}$, we also analyze the conductance $G(T)$ through the impurity, 


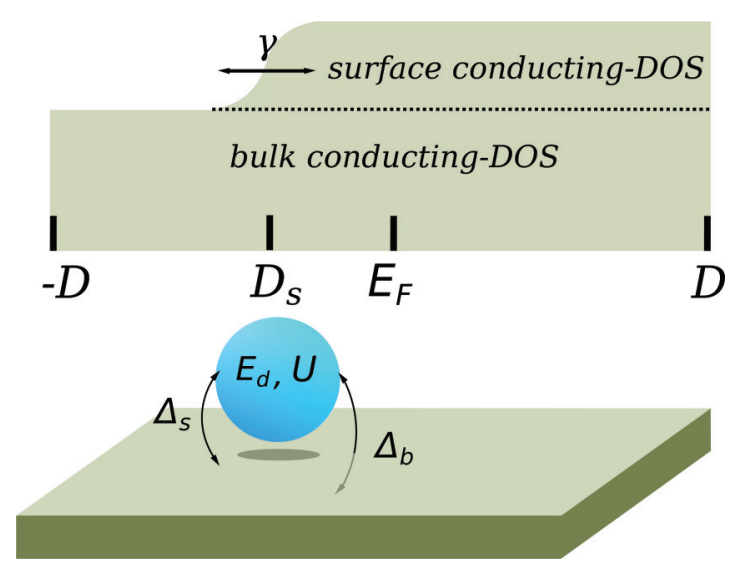

FIG. 1. Sketch of the setup. A magnetic impurity modeled as a single quantum dot with an energy level $E_{d}$ and Coulomb repulsion $U$ is deposited on the surface of a metal. The bulk density of the states extends from $-D$ to $D$, while the surface contribution abruptly starts at $D_{S}$. Both systems are coupled via the hybridization parameters $\Delta_{b}$ and $\Delta_{s} . E_{F}$ indicates the Fermi energy.

encountering strong suppression of the conductance at low temperatures when the onset approaches $E_{F}$. Finally, making contact with experiments, we study the conductance within the regime in which measurements by Limot et al. [5] and by Moro-Lagares et al. [16] were made.

Our results are important in order to predict deviations of the Kondo properties from their usual behaviors in systems where the onset of SDOS moves towards the Fermi energy.

This paper is organized as follows. In Sec. II we describe the theoretical model under study. Numerical results and their analysis are given in Sec. III. Finally, a summary and conclusions are presented in Sec. IV.

\section{MODEL}

The theoretical model we analyze in this paper, corresponding to the setup in Fig. 1, is defined by the following Hamiltonian:

$$
\begin{aligned}
H= & \sum_{k \sigma} \varepsilon_{k}^{s} s_{k \sigma}^{\dagger} s_{k \sigma}+\sum_{k \sigma} \varepsilon_{k}^{b} b_{k \sigma}^{\dagger} b_{k \sigma} \\
& +E_{d} \sum_{\sigma} n_{d \sigma}+U \sum d_{\uparrow}^{\dagger} d_{\uparrow} d_{\downarrow}^{\dagger} d_{\downarrow} \\
& +\sum_{k \sigma} V_{k}^{s}\left[d_{\sigma}^{\dagger} s_{k \sigma}+\text { H.c. }\right]+\sum_{k \sigma} V_{k}^{b}\left[d_{\sigma}^{\dagger} b_{k \sigma}+\text { H.c. }\right]
\end{aligned}
$$

where the first two terms correspond to surface and bulk noninteracting conduction electrons, respectively. The operator $d_{\sigma}^{\dagger}$ creates an electron with spin $\sigma$ at the magnetic impurity with $n_{d \sigma}=d_{\sigma}^{\dagger} d_{\sigma} . E_{d}$ and $U$ are the energy level and Coulomb repulsion, respectively. The last two terms describe the hybridization between the impurity and conduction electrons.

As usual, we describe the bulk contribution to conduction electrons with a constant density of states $\rho_{b}$ extended in a bandwidth from $-D$ to $D$. On the other hand, we model the surface contribution to the density of states including lifetime effects coming from quasiparticle interactions by a rounded- step function (see Fig. 1),

$$
\rho_{s}(\omega)=\frac{\rho_{s}}{\pi}\left\{\frac{\pi}{2}+\arctan \left[\left(\omega-D_{s}\right) /(\gamma / 2)\right]\right\} \theta(D-|\omega|),
$$

that starts at $D_{s}$ and is extended to $D$, where $\gamma$ represents the inverse lifetime of the surface states [5,24,25]. Regarding the upper limit of SDOS, it was found to be of the order of $1 \mathrm{eV}$ in $\mathrm{Au}(111)$ [2] and $0.2 \mathrm{eV}$ in $\mathrm{Ag}(111)$ [6]. In any case, we chose for simplicity the upper limit to coincide with the one of the bulk DOS $D$. This choice is justified because it does not modify the low-energy properties, near the Fermi level, which is the central point in our work. To provide an order of magnitude of $\rho_{b}$ and $\rho_{s}$, they were found to be $0.135 / \mathrm{eV}$ and $0.0466 / \mathrm{eV}$ in the case of $\operatorname{Ag}(111)$, respectively. [17]

The effect of the conduction bands on the impurity can be put in terms of an energy-dependent hybridization within the range $-D$ to $D$,

$$
\begin{aligned}
\Delta(\omega) & \equiv \pi \sum_{\nu k \sigma}\left|V_{k}^{v}\right|^{2} \delta\left(\omega-\varepsilon_{k}^{v}\right) \\
& =\Delta_{s} \tilde{\rho}_{s}(\omega)+\Delta_{b}
\end{aligned}
$$

where $\Delta_{v}=\pi\left|V^{v}\right|^{2} \rho_{v}$ and $\tilde{\rho}_{v}=\rho_{v}(\omega) / \rho_{\nu}$ is the bare density of states of the band electrons, normalized to its constant value.

The retarded Green's function of the impurity is given by

$$
G_{d \sigma}(z)=\frac{1}{z-E_{d}-\Sigma_{\Delta \sigma}(z)-\Sigma_{U \sigma}(z)},
$$

where $z=\omega+i 0$ and $\Sigma_{U \sigma}(z)$ and $\Sigma_{\Delta \sigma}(z)$ are the interacting (due to the Coulomb repulsion $U$ ) and the noninteracting [due to the one-body hybridization $\Delta(\omega)$ ] contributions to the selfenergy, respectively.

The noninteracting part of the self-energy is defined by the hybridization function

$$
\Sigma_{\Delta \sigma}(\omega)=\Gamma(\omega)-i \Delta(\omega),
$$

where the real part $\Gamma(\omega)$ can be obtained from $\Delta(\omega)$ by means of a Kramers-Kronig transformation.

As we mentioned in the Introduction, we solve the Hamiltonian in Eq. (1) by using the NRG technique. In addition to the usual $z$ averaging [26] and $\sigma$-trick [27] refinements when calculating thermodynamics and dynamical properties, NRG LJUBLJANA uses an improved discretization scheme [28] that allows the NRG method to handle energy-dependent hybridizations [25]. As usual, the half bandwidth of the bulk conduction electrons is taken as the unit of energy, $D=1$, and the Fermi energy is chosen to be $E_{F}=0$. Furthermore, we have used the renormalization parameter $\Lambda=2$ and a number of different values of $z, N_{z}=16$, in all NRG calculations.

\section{RESULTS}

\section{A. Sharp-step surface DOS}

We start this section by considering that the surface density of states can be modeled by a step function, that is, without the broadening introduced by the inverse lifetime, $\gamma=0$. This case was considered before for a fixed value of $\left|D_{s}\right| \gg T_{K}$ $[16,17,22,25,29]$. As we will show in this work, in the regime 
$\left|D_{s}\right| \ll T_{K}$ the step shape of SDOS contains the key ingredients related to the role of the onset in the Kondo physics.

\section{Kondo temperature as a function of $D_{s}$}

It will be instructive to present a brief summary of the main results of Ref. [22] regarding the variation of $T_{K}$ with $D_{s}$ for $\left|D_{s}\right| \gg T_{K}$. When the onset of the surface contribution to the conduction density of states is near the Fermi energy, the Kondo temperature as a function of $D_{s}$ is found to display a power law, $T_{K} \simeq C\left|D_{s}\right|^{\alpha}$, where the exponent $\alpha$ depends on the relative intensities between surface and bulk hybridizations with the impurity, $\Delta_{s} / \Delta_{b}$, and the ratio between on-site and Coulomb energies $E_{d} / U$ as well as the sign of $D_{s}$ and $C$ encloses the other dependences. This power law was obtained from a poor man's scaling (PMS) [30,31] approach to the effective Kondo model and confirmed numerically by using the noncrossing approximation (NCA) [30,32]. The validity of the above dependence applies for small values of $\left|D_{s}\right| \ll D$, but they are limited to $\left|D_{s}\right| \gg T_{K}$. Unfortunately, neither PMS nor NCA can describe the regime $\left|D_{s}\right| \leqslant T_{K}$. In the case of PMS, the renormalization group procedure ceases to be valid [30], while in the case of NCA, there are inaccurate results when dealing with small energies compared with the Kondo one [32].

Specifically, the Kondo scale was found to depend on the onset $D_{s}$ in the following way:

$$
\begin{gathered}
T_{K} \simeq A\left|D_{s}\right|^{\eta} D^{1-\eta} \exp \left[\frac{\pi E_{d}\left(E_{d}+U\right)}{2 U\left(\Delta_{b}+\Delta_{s}\right)}\right], \\
\eta=\frac{\Delta_{s}}{\left(\Delta_{b}+\Delta_{s}\right)}\left(1+\frac{E_{d}}{U}\right), \quad D_{s}<0,
\end{gathered}
$$

and

$$
\begin{aligned}
T_{K} & \simeq B\left(D_{s}\right)^{\zeta} D^{1-\zeta} \exp \left[\frac{\pi E_{d}\left(E_{d}+U\right)}{2 U \Delta_{b}}\right], \\
\zeta & =\frac{\Delta_{s} E_{d}}{\Delta_{b} U}, \quad D_{s}>0 .
\end{aligned}
$$

Both equations are valid in the limit of $\left|D_{s}\right| \gg T_{K}$ and when charge fluctuations are limited to only two configurations, $0 \leqslant$ $n_{d} \leqslant 1$ for $E_{d}<\infty, U \rightarrow \infty$ and $1 \leqslant n_{d} \leqslant 2$ for $E_{d}+U<$ $\infty,\left\{E_{d},-U\right\} \rightarrow-\infty$. The exponents $\eta$ and $\zeta$ were confirmed by NCA calculations for several values of the ratio $\Delta_{s} / \Delta_{b}$.

Within our NRG calculations, we get the Kondo temperature from the thermodynamic properties of the Hamiltonian according to Wilson's definition, $k_{B} T_{K} \chi_{\text {imp }}\left(k_{B} T_{K}\right) /\left(g \mu_{B}\right)^{2}=$ 0.07 , with $\chi_{\text {imp }}\left(k_{B} T\right)$ being the impurity contribution to the magnetic susceptibility as a function of temperature [33]. Throughout the rest of the paper we set $g \mu_{B}=1$ and $k_{B}=1$.

In Fig. 2 we show the results for $T_{K}$ as a function of $D_{s}$ in the selected case of $\Delta_{s}=\Delta_{b}$. The top panel displays the case with $U \rightarrow \infty$. From Eq. (6), a value of $\eta=1 / 2$ is expected. Fitting the NRG results with a power law of the form $A\left|D_{s}\right|^{\eta}$ for negative values of $D_{s}$, we obtain an exponent $\eta=0.49$. On the other hand, the bottom panel shows the results in the case of $E_{d}+U<\infty$ while $\left\{E_{d},-U\right\} \rightarrow-\infty$, for which an exponent $\zeta=-1$ for positive values of $D_{s}$ is expected. We obtain $\zeta=-0.94$ from the fitting. In both cases the accuracy of the fittings is confirmed by a correlation coefficient (cc) close to unity with an error of $10^{-4}$. The present results, also
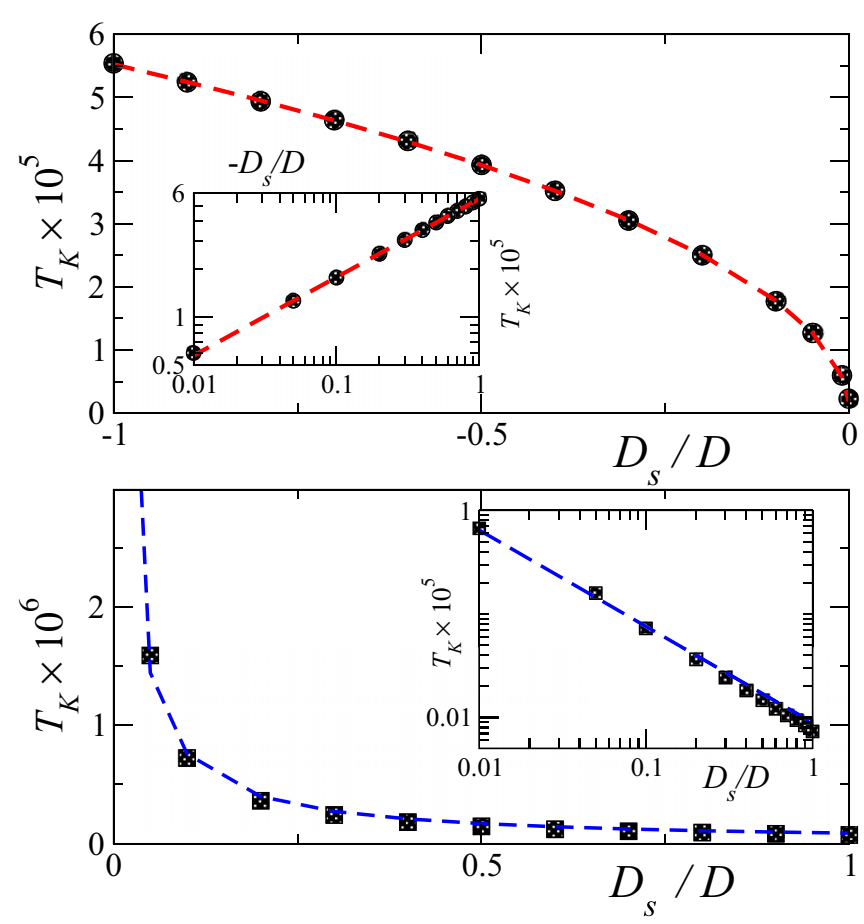

FIG. 2. Kondo temperature as a function of $D_{s}$ for $E_{d}=-0.04 D$, $U \rightarrow \infty$ (top panel) and $E_{d}+U=0.04 D,\left\{E_{d},-U\right\} \rightarrow-\infty$ (bottom panel). In both cases $\Delta_{b}=\Delta_{s}=0.5 D$. Similar values of $E_{d}$ and $E_{d}+U$ were used in Ref. [22]. Fitting parameters: $A=5.52 \times 10^{-5}$, $\eta=0.49, \mathrm{cc}=0.9999, B=8.9 \times 10^{-8}, \zeta=-0.94, \mathrm{cc}=0.9998$. The insets show the results on a logarithmic scale.

limited to $T_{K} \ll\left|D_{s}\right|$, verify the corresponding ones presented in Ref. [22]. Furthermore, other ratios of $\Delta_{s} / \Delta_{b}$ (not shown) were studied and also agree with the PMS and NCA results.

As we mentioned, the range $T_{K} \geqslant\left|D_{S}\right|$ is not accessible by either PMS or NCA. In Fig. 3 we show the values of $T_{K}$ when



FIG. 3. Kondo temperature as a function of $D_{s}$ for the same set of model parameters as in the top panel of Fig. 2. The dashed line stands for $D_{s}=0$, representing an energy scale $T^{*}=3.3 \times 10^{-7} D$. 


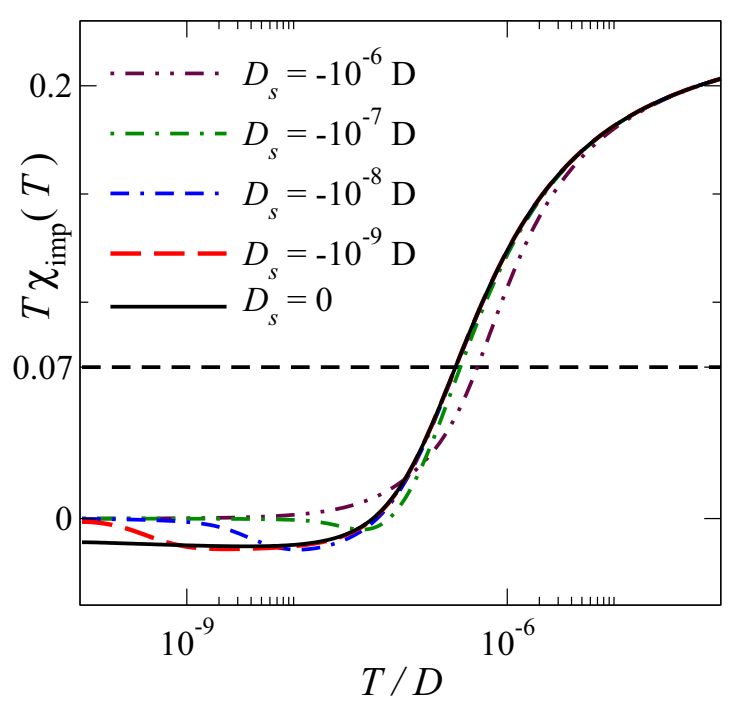

FIG. 4. Impurity magnetic susceptibility as a function of temperature for the same set of model parameters as in Fig. 3 and for different values of $D_{s} / D$. The black dashed solid line indicates the criteria for defining the Kondo temperature.

$D_{s}$ approaches the Fermi energy from negative values the in case of $U \rightarrow \infty$ and finite on-site energy $E_{d}$.

We can see from the plot that the scaling law in Eq. (6) breaks down as soon as $-D_{s}$ approaches $T_{K}$. Furthermore, $T_{K}$ seems to be constant in the range $\left|D_{s}\right| \ll T_{K}$ and saturates at a value $T^{*}$ when $D_{s}=0$ (dashed line). The corresponding values for positive $D_{s}$, although they do not obey any scaling, are present in order to show that a continuity function $T_{K}\left(D_{s}\right)$ is obtained when $D_{s}$ crosses the Fermi energy. Finally, we found (not shown) a similar deviation of the power law in the case of Eq. (7).

\section{Thermodynamic properties in the range $\left|D_{s}\right| \ll T_{K}$}

In what follows we turn our attention to the analysis of the Kondo physics through the impurity contribution to thermodynamic properties in the range $\left|D_{s}\right| \ll T_{K}$, in particular for $D_{s}=0$.

Interestingly, when $\left|D_{s}\right|<T_{K}$, the impurity magnetic susceptibility times temperature, $T \chi_{\text {imp }}(T)$, approaches zero from negative values very slowly. This unusual lowtemperature behavior is shown in Fig. 4 for the same set of model parameters as in Fig. 3. Notice that for finite values of $D_{s}$, the magnitude of $T \chi_{\text {imp }}(T)$ vanishes at sufficiently low temperature. In particular, for $D_{s}=0, T \chi_{\text {imp }}(T)$ approaches zero in the limit of $T \rightarrow 0$. From the results of Fig. 3, we do not expect a significant variation of the impurity properties for $D_{s}>0$.

When analyzing the thermodynamic properties of the impurity, we also notice that similar low-temperature features also appear in the impurity contribution to the entropy $S_{\text {imp }}(T)$. That is, $S_{\text {imp }}(T)$ goes to zero at low temperature from negative values (see top panel of Fig. 6).

We remind the reader that the impurity contributions to $\chi(T)$ and $S(T)$ are given by $\chi_{\mathrm{imp}}(T)=\chi_{\mathrm{tot}}(T)-\chi_{\mathrm{tot}}^{(0)}(T)$ and $S_{\text {imp }}(T)=S_{\text {tot }}(T)-S_{\text {tot }}^{(0)}(T)$, where $\chi_{\text {tot }}^{(0)}(T)\left[S_{\text {tot }}^{(0)}(T)\right]$ denotes

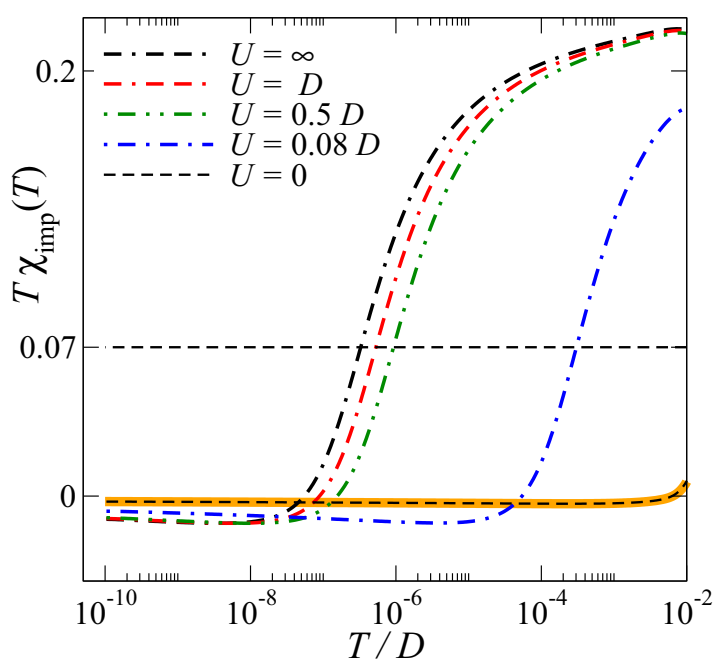

FIG. 5. Impurity magnetic susceptibility as a function of temperature in the case of $D_{s}=0$ and for different values of the Coulomb repulsion $U$ and for $E_{d}=-0.04 D$. The solid orange line corresponds to the analytic result for $U=0$ according to Eq. (8).

the system without impurity [33,34]. Therefore, even when $\chi_{\text {tot }}(T)\left[S_{\text {tot }}(T)\right]$ and $\chi_{\text {tot }}^{(0)}(T)\left[S_{\text {tot }}^{(0)}(T)\right]$ are separately positive, the impurity contribution can be negative.

One may wonder if the Coulomb repulsion plays any role in this behavior. In Fig. 5 we fix the onset of the surface density of states in $D_{s}=0$ and $E_{d}=-0.04 D$ and analyze $\chi_{\text {imp }}(T)$ for several values of $U$ from the noninteracting case $U=0$ to $U \rightarrow \infty$.

In fact, the negative values of entropy and magnetic susceptibility are already present for the non-interacting case $U=0$. The distinctive feature of the model Hamiltonian in Eq. (1) is enclosed in the one-body self-energy in Eq. (5), so in the following we analyze the magnetic susceptibility and entropy for the noninteracting model.

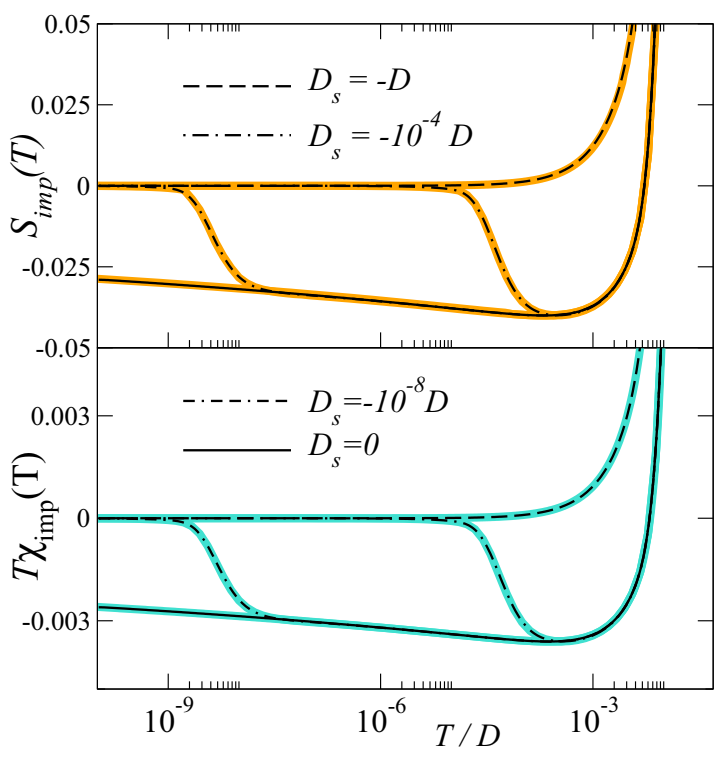

FIG. 6. Shown by thick lines, the $S_{\text {imp }}(T)$ (top panel) and $T \chi_{\text {imp }}(T)$ (bottom panel) results for $D_{s}=U=0$ from Eqs. (8). The thin black lines correspond to NRG calculations. 
The impurity magnetic susceptibility and entropy for the noninteracting version of the Hamiltonian in Eq. (1) are given by the following expressions:

$$
\begin{aligned}
S_{\text {imp }}(T) & =\frac{2}{\pi T} \operatorname{Im} \int_{-\infty}^{+\infty} d \omega f^{\prime}(\omega) \omega \ln G_{0 d}^{-1}(\omega), \\
T \chi_{\text {imp }}(T) & =\frac{1}{2 \pi} \operatorname{Im} \int_{-\infty}^{+\infty} d \omega f^{\prime}(\omega)[1-2 f(\omega)] \ln G_{0 d}^{-1}(\omega),
\end{aligned}
$$

where $G_{0 d}^{-1}(\omega)=\omega-E_{d}-\Sigma_{\Delta}(\omega)$ is the noninteracting electron impurity Green's function independent of the spin.

Both magnitudes were calculated in the case of $D_{s}=0$, and the results as a function of temperature are displayed in Fig. 6, represented by solid lines. Clearly, the same features as in Figs. 4 and 5 are present. Although it is not necessary, we take the opportunity to benchmark the NRG when calculating the same thermodynamic impurity contribution for $U=0$. As is clear from Fig. 6, the results obtained are on top of the analytical ones. This accuracy when dealing with energydependent hybridizations is due to the improved discretization scheme detailed in Ref. [28].

The low-temperature behavior of Eq. (8) is given by the corresponding low-energy form of the one-body self-energy, which in the limit of $D_{s}=0$ is

$$
\Sigma_{\Delta}(\omega) \sim \frac{\Delta_{s}}{\pi} \ln \left|\frac{\omega}{D}\right|-i \Delta(\omega) .
$$

Note the divergent form at low energies. Then, the impurity entropy and magnetic susceptibility become

$$
\begin{gathered}
S_{\mathrm{imp}}(T) \sim-\frac{(1 / 2) \ln 4}{\ln (1 / 2 T)}+O\left(T^{2}\right), \\
T \chi_{\mathrm{imp}}(T) \sim-\frac{(1 / 8)}{\ln (1 / 2 T)}+O\left(T^{2}\right) .
\end{gathered}
$$

Remarkably, the usual Fermi liquid properties for $\left|D_{s}\right| \leqslant T_{K}$ are recovered at low enough temperature, and for the special case in which the onset of the surface DOS coincides with the Fermi energy, they are reached logarithmically slowly.

Here we would like to mention the work of Mitchell and Fritz [35], in which they analyzed the Kondo effect in graphene with vacancies. In spite of the two very different systems, the one studied by Mitchell and coworkers and the present one, they also found negative impurity contributions to magnetic susceptibility and entropy at low temperatures independent of the value of the Coulomb repulsion. In their case, the cause of such behavior was attributed to a logarithmically divergent imaginary part of the one-body hybridization $\Sigma_{\Delta}$ coming from a zero mode in graphene with defects. In our case, we have a logarithmically divergent real part of the one-body hybridization coming from a nonanalyticity in the conduction DOS. In any case, both hybridization shapes give rise to similar low-temperature features in the impurity properties.

\section{Spectral density for $D_{s}=0$}

Since $\Sigma_{\Delta}(\omega)$ becomes infinite for $D_{s}=\omega=0$, it is expected that the impurity spectral density, $\rho_{0 d}(\omega)=$ $-\operatorname{Im} G_{0 d}(\omega) / \pi$, vanishes at the Fermi energy. This is explic-

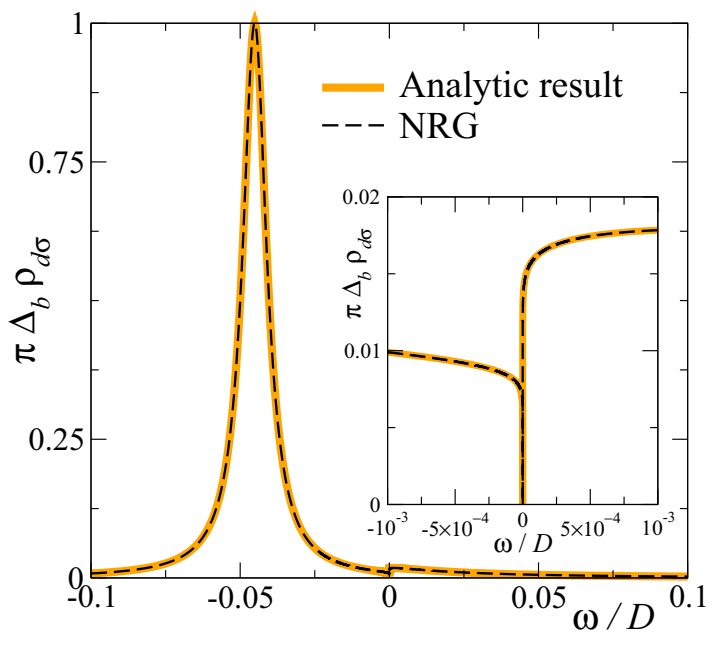

FIG. 7. Noninteracting impurity spectral density $\rho_{0 d}(\omega)$ for $D_{s}=$ 0 obtained from Eq. (4) with $\Sigma_{U \sigma}(z)=0$ and NRG with $U=0$. Other parameters are as in Fig. 2.

itly shown in Fig. 7, in which both the analytical and NRG calculations are again on top of each other.

As we have shown, the Coulomb repulsion is not responsible for this effect, so one may wonder in what way this logarithmic energy dependence affects the Kondo effect. Once the interaction $U$ is turned on and the temperature is lowered, the spectral weight at the Fermi level should be increased due to the appearance of the Kondo peak [30]. Therefore, competition between the effects is expected. In Fig. 8 the spectral density is shown for two selected values of the Coulomb repulsion, $U=-2 E_{d}$ and $U=\infty$, and for $D_{s}=0$. The NRG calculations were done at $T=10^{-3} T_{K}$, with $T_{K}$ being the corresponding one for each value of $U$ according to Fig. 5. Several aspects can be discussed from these results. In the first place, the top panel shows the spectral density in its extended frequency region. The solid line has a value of the Coulomb interaction in such a way that $2 E_{d}+U=0$, which is the usual condition for particle-hole symmetry. This symmetry is clearly broken due to the asymmetry in the hybridization function in Eq. (3) (see also the cartoon of the conduction DOS in Fig. 1). While the lower charge transfer peak, located at energy $\omega \sim E_{d}$, has a width of the order of $4 \Delta_{b}[36,37]$, the upper one, located at $\omega \sim E_{d}+U$, has a width of the order of $4\left(\Delta_{b}+\Delta_{s}\right)$, and as a consequence, its intensity is reduced. The $U=\infty$ case has a similar width of $4 \Delta_{b}$ in the unique charge transfer peak. The central peak corresponds to the Kondo resonance. In a manner similar to how the width of the Fano-Kondo antiresonance is related to $T_{K}$, the width of the Kondo is also related to $T_{K}$. In relation to the low-energy features of the spectral density, the bottom panel of Fig. 8 shows a detail of the Kondo resonance. Once the energy dependence in both curves is shown in units of $T_{K}$, they are quite similar as a consequence of the universality of the Kondo phenomena.

The logarithmic divergence of the one-body hybridization splits the Kondo resonance into two pieces around the Fermi level. Furthermore, suppression of the spectral weight at $\omega=$ $E_{F}$ is observed independent of the value of the Coulomb 

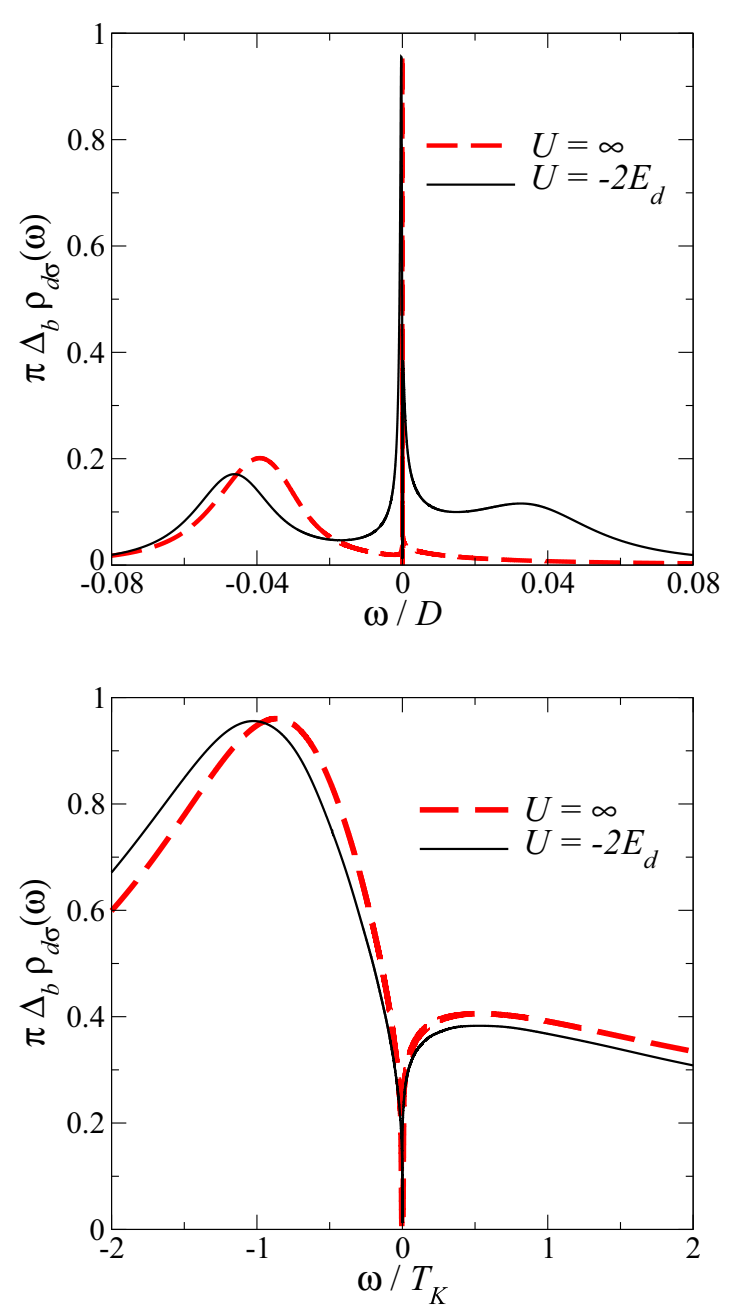

FIG. 8. Top: spectral densities $\rho_{d \sigma}$ as a function of frequency for $D_{s}=0$ and $U=-2 E d$ (solid line) and $U=\infty$ (dashed line). $E_{d}=$ -0.04 and $\Delta_{s}=\Delta_{b}=0.005$. Bottom: low-energy region in units of $\omega / T_{K}$ with $T_{K}(U=-2 E d)=3.04 \times 10^{-4} D$ and $T_{K}(U=\infty)=$ $3.40 \times 10^{-7} \mathrm{D}$. Calculations were done at $T=10^{-3} T_{K}$.

repulsion. Note that for negative energies close to the Fermi one, the Kondo peak seems to agree with the usual Friedel sum rule $\rho_{d \sigma}(0)=1 / \pi \Delta$, with $\Delta=\Delta_{b}$. On the other hand, since the SDOS is already turned on for positive energies, the hole contribution of the Kondo resonance, $\omega \gtrsim 0$, seems to be related to the total contribution $\Delta=\Delta_{b}+\Delta_{s}$. We have confirmed the last statement by plotting in Fig. 9 the spectral density for another relation between $\Delta_{b}$ and $\Delta_{s}$. In particular we choose the ratio $\Delta_{s} / \Delta_{b}=1 / 4$, which has been found to be the lower limit for the surface contribution to the total hybridization in Co impurities deposited on the $\operatorname{Ag}(111)$ surface [16]. What we observed from the plot is that while the negative portion of the Kondo resonance approaches 1 when scaled by $\pi \Delta_{b}$, the positive one reaches the same value when it is scaled by $\pi\left(\Delta_{b}+\Delta_{s}\right)$. Note that for the selected parameters the Kondo resonance is slightly asymmetric with more weight in the negative region, so it is closer to 1 for negative energies than for the positive ones.

In any case, the usual Friedel sum rule does not apply since $\rho_{d \sigma}(0) \sim 0$. Instead, as we will see, the weight of the spectral

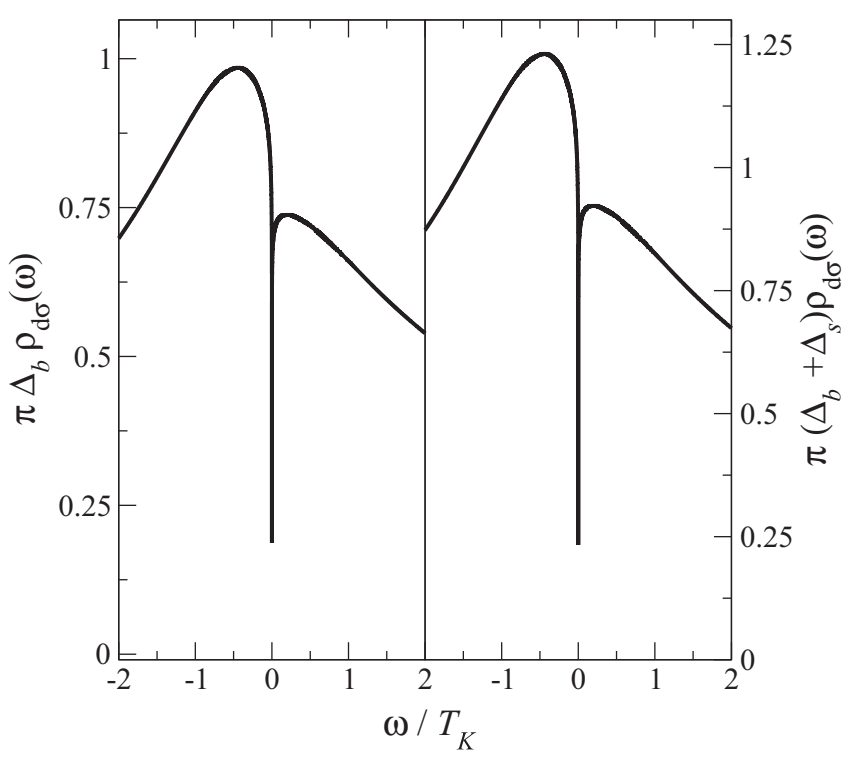

FIG. 9. Spectral density $\rho_{d \sigma}$ in the low-energy region in units of $\omega / T_{K}\left(T_{K}=3 \times 10^{-5} D\right)$ for $D_{s}=0$ and $\Delta_{s}=0.25 \Delta_{b}=0.00125$. $U=-2 E d$ with $E_{d}=-0.04$. Left: $\rho_{d \sigma}$ scaled by $\pi \Delta_{b}$. Right: $\rho_{d \sigma}$ scaled by $\pi\left(\Delta_{b}+\Delta_{s}\right)$.

density at the Fermi energy is consistent with the generalized Friedel sum rule [38,39].

\section{Conductance through the impurity and occupation}

The reduction in the spectral weight at low energies affects the transport properties of the model. The equilibrium conductance $G(T)$ directly depends on $\rho_{d \sigma}(\omega)$ [40],

$$
G(T)=G_{0} \frac{\pi}{2} \sum_{\sigma} \int_{-\infty}^{+\infty} d \omega\left[-f^{\prime}(\omega)\right] \Delta(\omega) \rho_{d \sigma}(\omega),
$$

where $G_{0}=2 e^{2} / h$ is the quantum of conductance. In particular, a strong reduction in the equilibrium conductance $G(T)$ at low temperatures is expected. In Fig. 10 we show the NRG calculations for the conductance as a function of temperature. In the top panel we fix $U+2 E_{d}=0$ with $U=0.08 D$ while varying the values of the onset $D_{s}$. When $D_{s}=-D$, the surface contribution to the hybridization agrees with the bulk one, and the conductance displays the usual behavior reaching the maximum value for this problem of $G_{0}$ for temperatures $T \ll T_{K}$. For smaller values of $\left|D_{s}\right|$ that are still large compared with the corresponding $T_{K}$, the shape of the conductance is not affected. However, when $-D_{s}$ is of the order of $T_{K}$ $\left(\sim 10^{-4} D\right), G(T)$ saturates at a reduced fraction of $G_{0}$. The reduction of the saturated conductance grows as $D_{s}$ approaches zero. In the limiting case of $D_{s}=0$, the conductance vanishes at low temperatures in a logarithmic shape.

In the bottom panel of Fig. 10 we show the obtained values of $G(T)$ for $D_{s}=0$ and two different values of the Coulomb repulsion, $U+2 E_{d}=0$ with $U=0.08 D$ (black solid curve) and $U \rightarrow \infty$ (red dashed line). As is clear from the results, when scaling the temperatures by the corresponding Kondo one, both curves display an identical temperature dependence, as expected from the universality of the Kondo effect. 


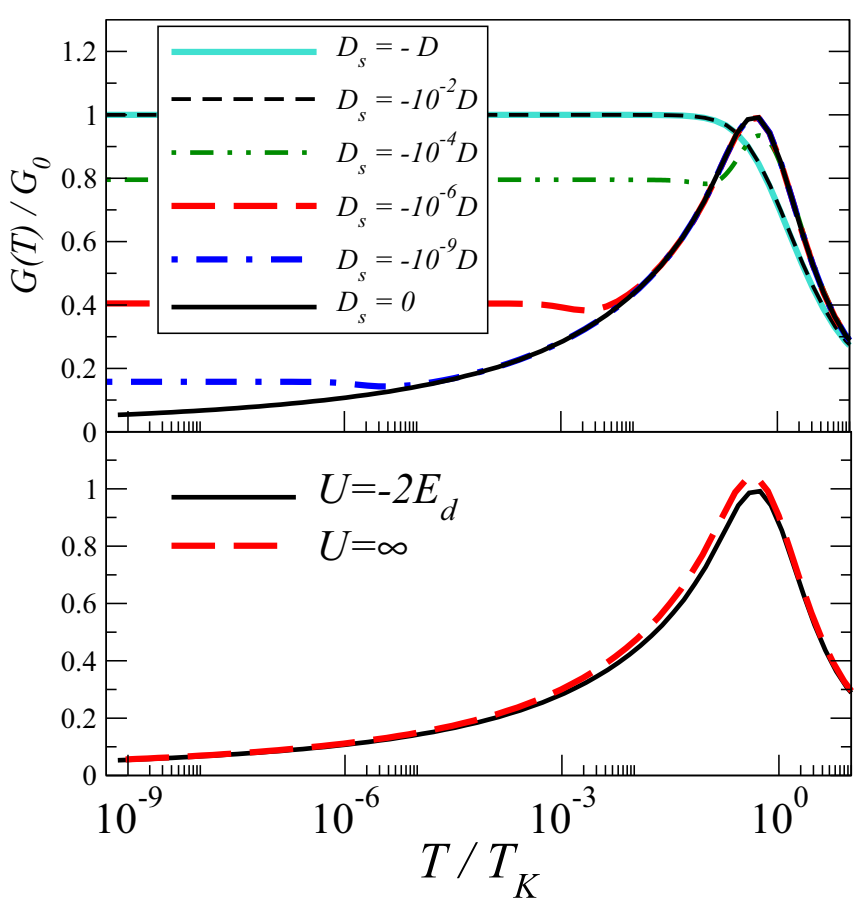

FIG. 10. Equilibrium conductance in units of $G_{0}=2 e^{2} / h$ as a function of temperature in units of $T_{K}$. Top: Several values of $D_{s}$ for the case $U+2 E_{d}=0$ with $U=0.08 D$. Bottom: $D_{s}=0$ for the cases $U+2 E_{d}=0$ with $U=0.08 D$ (black solid curve) and $U \rightarrow \infty$ (red dashed line).

The results of the intermediate plateaus in the conductance can be understood in terms of the generalized Friedel sum rule [38,39],

$$
\begin{aligned}
G(T) / G_{0} & =\sin ^{2}\left[\frac{\pi}{2}\left(n_{\mathrm{imp}}-n_{c}\right)\right] \\
& =\pi\left(\Delta_{b}+\Delta_{s}\right) \rho_{d \sigma}(0),
\end{aligned}
$$

where $n_{\mathrm{imp}}$ is the impurity occupation and

$$
n_{c}=-\operatorname{Im} \sum_{\sigma} \int_{-\infty}^{E_{F}} \frac{d \omega}{\pi} G_{d \sigma}(\omega) \frac{\partial \Delta(\omega)}{\partial \omega}
$$

is related to the change in the charge in the conduction band as a consequence of the presence of the impurity. In the usual case of a flat hybridization, this term vanishes due to $\frac{\partial \Delta(\omega)}{\partial \omega} \sim 0$. However, in our case we expect its influence to be rather large when $D_{s} \sim E_{F}$. For the noninteracting model, we evaluate Eq. (13), and together with the results of the impurity occupation, Eq. (12) can be verified. In the top panel of Fig. 11 we show the results of $n_{c}$ and $n_{\text {imp }}$ at zero temperature as a function of $D_{s}$ in the case of $U+2 E_{d}=0$ with $U=0$. While the impurity population is rather constant, it is clear that $n_{c}$ approaches $n_{\text {imp }}$ as $\left|D_{s}\right|$ is decreased. Therefore, both $G(T \rightarrow 0)$ and $\rho_{d \sigma}(\omega \rightarrow 0)$ vanish when $D_{s} \rightarrow 0$. In the bottom panel of Fig. 11 we show the NRG results for $n_{c}$ and $n_{\text {imp }}$ at temperature $T=10^{-3} T_{K}$ as a function of $D_{s}$ in the case of $U+2 E_{d}=0$ with $U=0.08 D$. As we have previously mentioned, particle-hole symmetry is slightly broken (see Fig. 8), and therefore, the impurity occupation is very close to, but not exactly, 1 . However, $n_{c}$ strongly changes from 0 $\left(D_{s}=-D\right)$ to $1\left(D_{s}=0\right)$. In this case, when interactions are



FIG. 11. $n_{c}$ and $n_{\text {imp }}$ as a function of $D_{s}$ for $U+2 E_{d}=0$. Top: $U=0$. Bottom: $U=0.08 D$. In both cases we have used $\Delta_{s}=\Delta_{b}=$ $0.005 D$.

turning on, we obtain $n_{c}$ not directly from Eq. (13) but from the more accurate values of the low-temperature conductance according to Eq. (12). Note that in the case of $D_{s}=0$ the result $n_{c}=n_{\text {imp }}$ is reached only asymptotically for $T \rightarrow 0$ (see Fig. 10).

\section{B. Rounded-step surface DOS}

We end the present work by considering finite-lifetime effects $\gamma$ leading to a smoother onset of the band edge, as shown in Eq. (2). This is especially important to make contact with real systems, in particular with the experiment of Limot and coworkers [5] in which the authors performed scanning tunneling spectroscopy measurements of magnetic (Co) and nonmagnetic (Ag) atoms on $\mathrm{Ag}(111)$ and $\mathrm{Cu}(111)$ and analyzed a bound state that appears after impurity deposition near the onset of SDOS. In the case of $\mathrm{Co} / \mathrm{Ag}(111)$ and $\mathrm{Co} / \mathrm{Cu}(111)$ a dip corresponding to a Fano-Kondo antiresonance appears at the Fermi level. For a quantitative analysis of the experiment, the authors modeled the surface DOS by including lifetime effects.

Therefore, it is interesting to see how rapidly the logarithmic shapes of the analyzed properties in this paper blur in the presence of $\gamma$. When a Co atom is deposited on a clean $\operatorname{Ag}(111)$ surface, the measure $T_{K} \sim 83 \mathrm{~K}$ is found, and the data are adjusted by $\gamma=7 \mathrm{meV}=81.2 \mathrm{~K}$, and therefore, $\gamma \sim T_{K}$. On the other hand, when a Co atom is deposited on $\mathrm{Cu}(111)$, the values are $T_{K} \sim 63 \mathrm{~K}$ and $\gamma=24 \mathrm{meV}=$ $278.4 \mathrm{~K}$, which leads to the ratio $\gamma / T_{K} \sim 4.4$.

In Fig. 12 we show the NRG results for the conductance as a function of temperature when the surface hybridization is given by Eq. (2) and for several values of the ratio $\gamma / T_{K}$. Here, we do not expect to make a quantitative description of each system mentioned above but provide an analysis of what the effect of $\gamma$ will be given the measured values of $T_{K}$. To this end, we have chosen a generic set of model 


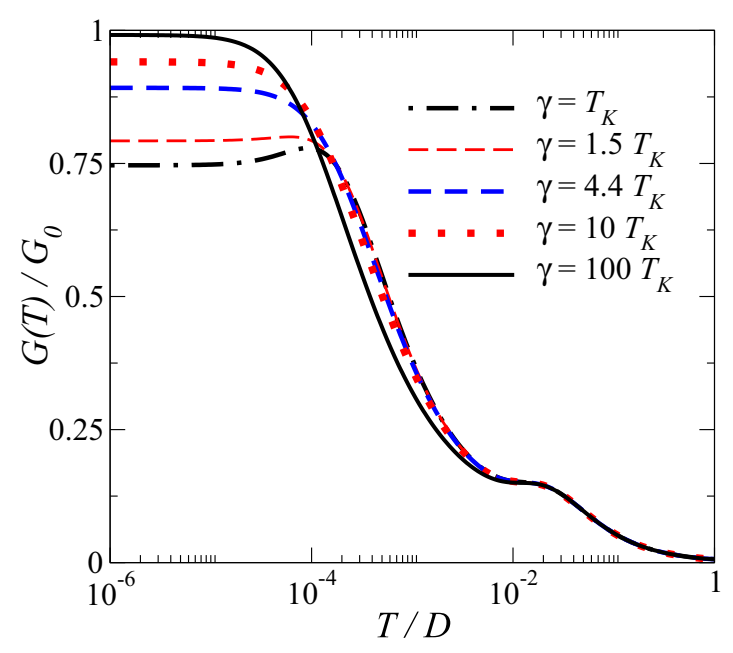

FIG. 12. Conductance as a function of temperature in the case of $D_{s}=0$ for several values of the inverse lifetime $\gamma$ in units of $T_{K}$. As a reference, we use $T_{K}$ corresponding to the black solid line in Fig. 8. $\gamma=T_{K}$ and $\gamma=4.4 T_{K}$ represent $\mathrm{Co} / \mathrm{Ag}(111)$ and $\mathrm{Co} / \mathrm{Cu}(111)$, respectively, in Limot et al.'s experiment, while $\gamma=$ $1.5 T_{K}$ represents $\mathrm{Co} / \mathrm{Ag}(111)$ in Moro-Lagares et al.'s experiment.

parameters, $E_{d}, U, \Delta_{b}$, and $\Delta_{s}$, and have compared the effect of $\gamma$ against the obtained $T_{K}$. Provided that in the Kondo regime, the only relevant energy scale is, in fact, $T_{K}$ and all magnitudes display a universal dependence when scaled by it, for instance, the spectral density in Fig. 8 (bottom panel) and the conductance in Fig. 10, the results in Fig. 12 are quite general. The case of $\gamma / T_{K}=1$ represents $\mathrm{Co} / \mathrm{Ag}(111)$ in Limot $e$ t al.'s experiment. Clearly, when the onset of the surface states coincides with the Fermi energy, even in the case of having a lifetime in the DOS, the conductance is reduced significantly, near a factor of $25 \%$ of the usual ideal value of $G_{0}$. The case of $\gamma / T_{K}=4.4$ represents $\mathrm{Co} / \mathrm{Cu}(111)$ with also an important reduction in the low-temperature conductance. For comparison, we also show the case $\gamma / T_{K}=10$, for which the effect of the step is still present. Only when $\gamma / T_{K} \geqslant 100$ is the conducting DOS smooth enough to get $n_{c} \sim 0$ and can the ideal conductance be reached. Note, however, that here we do not include other degrees of freedom in the system that contribute to enlarging the inverse lifetime in the SDOS. Such contributions should be included in order to fully analyze the Kondo properties.

In addition to Limot et al.'s experiment, that of Moro-Lagares and coworkers [16] studied the Kondo phenomenon that appears after deposition of a Co atom on a clean $\operatorname{Ag}(111)$ surface. In that work, the main result is a quantification of the role of the surface state in the Kondo effect and provides a lower bound for the ratio of hybridizations $r_{s b}=\Delta_{s} / \Delta_{b}=1 / 4$.

Regarding $T_{K}$, the Fano-Kondo line shape was also analyzed, and they found $T_{K}=52 \mathrm{~K}$, which increases the ratio $\gamma / T_{K} \sim 1.56$ compared with that in Limot et al.'s work. The discrepancy between the Kondo temperature estimated by Limot et al. and Moro-Lagares et al. is explained in Ref. [41] and will not be discuss here. For this ratio between $\gamma$ and $T_{K}$, the low-temperature plateau in the conductance is reduced by nearly $20 \%$.

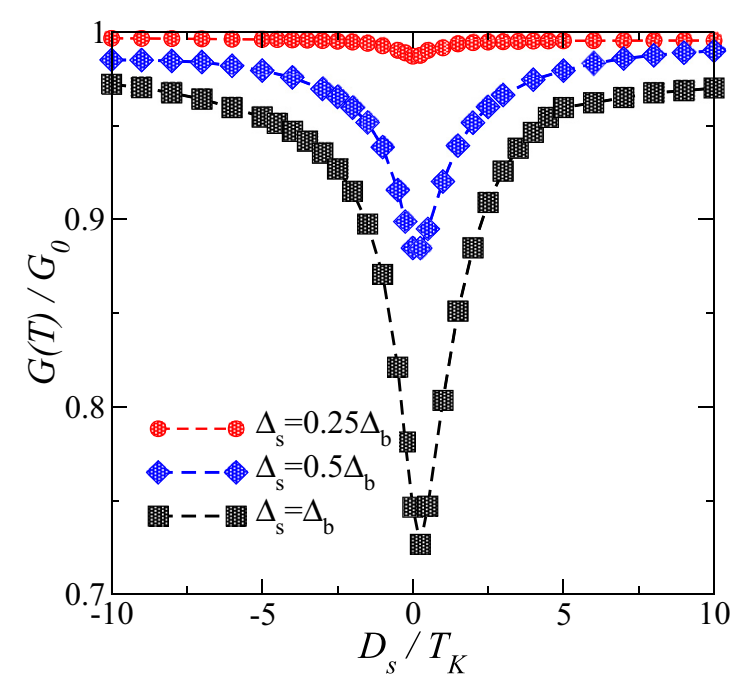

FIG. 13. Saturated conductance extracted from the low-temperature plateaus in Fig. 12 as a function of $D_{s}$ for several values of the ratio $r_{s b}=\Delta_{s} / \Delta_{b} . \gamma=T_{K}$ as $\mathrm{Co} / \operatorname{Ag}(111)$ in Limot et al.'s experiment, where $T_{K}$ corresponds to the black solid line in Fig. 8.

In any case, the low-temperature dependence of $G(T)$ is modified by the presence of the onset $D_{s}$ even if a nonvanishing inverse lifetime $\gamma$ is incorporated when describing the surface conducting electrons.

As another way to observe the influence on transport measurements when moving the onset of SDOS, we plot in Fig. 13 the saturated conductance as a function of $D_{s}$. When $\gamma=T_{K}$, such as $\mathrm{Co} / \mathrm{Ag}(111)$ in Limot et al.'s experiment, a dip centered at $D_{s}=0$ is clearly visible. The intensity of the dip depends on the ratio $r_{s b}$. Increasing $r_{s b}$ results in an increment in the dip intensity. Once $\left|D_{s}\right| \gg T_{K}$, the conductance approach $G_{0}$ independently of $r_{s b}$. Regarding the values of the ratio $r_{s b}$, Žitko [25] argued in favor of $r_{s b}=1$, while the experiment of Moro-Lagares et al., as we have mentioned, results in $r_{s b} \geqslant 1 / 4$. Note that from the results shown if Fig. 10, for $\gamma=0$ the dip reaches its maximum intensity, $G\left(D_{s}=0\right) \rightarrow 0$, independent of $r_{s b}$. Therefore, for all realistic values of $r_{s b}$, even if $\gamma$ is present, evidence of the conductance through the impurity when $D_{s}$ crosses the Fermi level should be present.

\section{SUMMARY}

We have analyzed thermodynamic and dynamical properties of a magnetic adatom deposited on a metal surface which contains a rounded step in the surface contribution to the total density of states of conduction electrons. The model represents realistic setups for magnetic adatoms, like $\mathrm{Co}$, on a (111) surface of $\mathrm{Cu}, \mathrm{Ag}$, and $\mathrm{Au}$. In such metals, a twodimensional surface band (Shockley states) starts at an energy $D_{s}$ moderately below the Fermi level. As we mentioned in the Introduction, $D_{s}$ can move continuously.

Within this framework, we have studied the lowtemperature properties inside the Kondo regime as a function of $D_{s}$, in particular when it crosses $E_{F}$. 
First, we confirmed by means of exact numerical renormalization group calculations the power law Kondo temperature dependence on $D_{s}$ found recently by using poor man's scaling arguments and noncrossing approximation calculations in Ref. [22]. After that, we focused on the physics that emerges from $D_{s}=0$. We investigated several magnitudes of interest, like the impurity contribution to magnetic susceptibility and entropy, spectral density, and equilibrium conductance. At all magnitudes, the step in the surface DOS plays an important role when $D_{s}$ approaches $E_{F}$. Interestingly, the magnetic susceptibility and entropy as a function of temperature exhibit negative values and go to zero slowly in a logarithmic shape. This result becomes independent of the impurity parameters such as the Coulomb repulsion already present in the noninteracting case.

Second, we examined the impurity spectral density as well as the conductance. Both are strongly affected by $D_{s}$. The results were interpreted in terms of the generalized Friedel sum rule in which the change in the charge in the conduction band $n_{c}$ strongly depends on $D_{s}$.

Finally, we emphasized the relevance of our study not only for academic reasons but also for real experiments. We analyzed the situation in the experiment of Limot et al. [5] and also in the one performed by Moro-Lagares and coworkers [16].

In both experiments a dip in $d I / d V$, representing the Fano antiresonance, was found at bias voltage $V=0$. The dip is a clear manifestation of the Kondo resonance in the spectral density of Co. Although the results in Fig. 8 represent the limiting case of $D_{s} \sim 0$, the shape and intensity of such dips are already affected by the step in the surface DOS even for finite values of $D_{s}$. In particular, we found that, if $D_{s}$ moves towards $E_{F}$, measurable effects emerge. When the low-temperature conductance is plotted as a function of $D_{s}$, which can now be experimentally done [10], we show that a dip should be present around $D_{s} \sim 0$. Therefore, we expect that our work will stimulate further research on the area of a magnetic adatom on metallic surfaces.

\section{ACKNOWLEDGMENTS}

We thank A. A. Aligia, L. O. Manuel, and I. Hamad for useful comments and careful reading of the manuscript. This work was sponsored by PIP 364 of CONICET and PICT 20172726 of ANPCyT (Argentina).
[1] J. P. Gauyacq, A. G. Borisov, and A. K. Kazansky, Impurityinduced localization of the $2 \mathrm{D}$ surface-state continuum on a metal surface, Appl. Phys. A 78, 141 (2004).

[2] H. Ibach, Physics of Surfaces and Interfaces (Springer, Berlin, 2006).

[3] N. Knorr, M. A. Schneider, L. Diekhöner, P. Wahl, and K. Kern, Kondo Effect of Single Co Adatoms on Cu Surfaces, Phys. Rev. Lett. 88, 096804 (2002).

[4] H. Cercellier, C. Didiot, Y. Fagot-Revurat, B. Kierren, L. Moreau, D. Malterre, and F. Reinert, Interplay between structural, chemical, and spectroscopic properties of $\mathrm{Ag} / \mathrm{Au}(111)$ epitaxial ultrathin films: A way to tune the Rashba coupling, Phys. Rev. B 73, 195413 (2006).

[5] L. Limot, E. Pehlke, J. Kröger, and R. Berndt, SurfaceState Localization at Adatoms, Phys. Rev. Lett. 94, 036805 (2005).

[6] S. Liu, A. Shiotari, D. Baugh, M. Wolf, and T. Kumagai, Enhanced resolution imaging of ultrathin $\mathrm{ZnO}$ layers on $\mathrm{Ag}(111)$ by multiple hydrogen molecules in a scanning tunneling microscope junction, Phys. Rev. B 97, 195417 (2018).

[7] K. Morgenstern, K.-F. Braun, and K.-H. Rieder, Surface-State Depopulation on Small Ag(111) Terraces, Phys. Rev. Lett. 89, 226801 (2002).

[8] G. Neuhold and K. Horn, Depopulation of the Ag(111) Surface State Assigned to Strain in Epitaxial Films, Phys. Rev. Lett. 78, 1327 (1997).

[9] Z. M. Abd El-Fattah, M. Matena, M. Corso, M. Ormaza, J. E. Ortega, and F. Schiller, Modifying the Cu (111) Shockley surface state by Au alloying, Phys. Rev. B 86, 245418 (2012), and references therein.

[10] C. W. Hicks, M. E. Barber, S. D. Edkins, D. O. Brodsky, and A. P. Mackenzie, Piezoelectric-based apparatus for strain tuning, Rev. Sci. Instrum. 85, 065003 (2014).
[11] C. W. Hicks, D. O. Brodsky, E. A. Yelland, A. S. Gibbs, J. A. N. Bruin, M. E. Barber, S. D. Edkins, K. Nishimura, S. Yonezawa, Y. Maeno, and A. P. Mackenzie, Strong increase of $T_{c}$ of $\mathrm{Sr}_{2} \mathrm{RuO}_{4}$ under both tensile and compressive strain, Science 344, 283 (2014).

[12] J. Li, W.-D. Schneider, R. Berndt, and B. Delley, Kondo Scattering Observed at a Single Magnetic Impurity, Phys. Rev. Lett. 80, 2893 (1998).

[13] V. Madhavan, W. Chen, T. Jamneala, M. F. Crommie, and N. S. Wingreen, Tunneling into a single magnetic atom: Spectroscopic evidence of the Kondo resonance, Science 280, 567 (1998).

[14] H. C. Manoharan, C. P. Lutz, and D. M. Eigler, Quantum mirages formed by coherent projection of electronic structure, Nature (London) 403, 512 (2000).

[15] D. Serrate, M. Moro-Lagares, M. Piantek, J. I. Pascual, and M. R. Ibarra, Enhanced hydrogen dissociation by individual Co atoms supported on Ag (111), J. Phys. Chem. C 118, 5827 (2014).

[16] M. Moro-Lagares, J. Fernández, P. Roura-Bas, M. R. Ibarra, A. A. Aligia, and D. Serrate, Quantifying the leading role of the surface state in the Kondo effect of Co/Ag(111), Phys. Rev. B 97, 235442 (2018).

[17] J. Fernández, M. Moro-Lagares, D. Serrate, and A. A. Aligia, Manipulation of the surface density of states of $\operatorname{Ag}(111)$ by means of resonators: Experiment and theory, Phys. Rev. B 94, 075408 (2016).

[18] Q. L. Li, C. Zheng, R. Wang, B. F. Miao, R. X. Cao, L. Sun, D. Wu, Y. Z. Wu, S. C. Li, B. G. Wang, and H. F. Ding, Role of the surface state in the Kondo resonance width of a Co single adatom on Ag(111), Phys. Rev. B 97, 035417 (2018).

[19] R. Hiraoka, E. Minamitani, R. Arafune, N. Tsukahara, S. Watanabe, M. Kawai, and N. Takagi, Single-molecule quantum dot as a Kondo simulator, Nat. Commun. 8, 16012 (2017). 
[20] E. Minamitani, N. Tsukahara, D. Matsunaka, Y. Kim, N. Takagi, and M. Kawai, Symmetry-Driven Novel Kondo Effect in a Molecule, Phys. Rev. Lett. 109, 086602 (2012).

[21] O. Újsághy, J. Kroha, L. Szunyogh, and A. Zawadowski, Theory of the Fano Resonance in the STM Tunneling Density of States due to a Single Kondo Impurity, Phys. Rev. Lett. 85, 2557 (2000).

[22] J. Fernández, A. A. Aligia, P. Roura-Bas, and J. A. Andrade, Kondo temperature when the Fermi level is near a step in the conduction density of states, Phys. Rev. B 95, 045143 (2017).

[23] R. Zitko, NRG LJUBLJANA, open-source numerical renormalization group code, http://nrgljubljana.ijs.si.

[24] J. Li, W.-D. Schneider, R. Berndt, O. R. Bryant, and S. Crampin, Surface-State Lifetime Measured by Scanning Tunneling Spectroscopy, Phys. Rev. Lett. 81, 4464 (1998).

[25] R. Žitko, Numerical renormalization group calculations of ground-state energy: Application to correlation effects in the adsorption of magnetic impurities on metal surfaces, Phys. Rev. B 79, 233105 (2009).

[26] H. O. Frota and L. N. Oliveira, Photoemission spectroscopy for the spin-degenerate Anderson model, Phys. Rev. B 33, 7871 (1986); V. L. Campo and L. N. Oliveira, Alternative discretization in the numerical renormalization-group method, ibid. 72, 104432 (2005).

[27] R. Žitko and T. Pruschke, Energy resolution and discretization artifacts in the numerical renormalization group, Phys. Rev. B 79, 085106 (2009).

[28] R. Žitko, Adaptive logarithmic discretization for numerical renormalization group methods, Comput. Phys. Commun. 180, 1271 (2009).

[29] A. A. Aligia and A. M. Lobos, Mirages and many-body effects in quantum corrals, J. Phys.: Condens. Matter 17, S1095 (2005).

[30] A. C. Hewson, The Kondo Problem to Heavy Fermions (Cambridge University Press, Cambridge, 1997).
[31] P. W. Anderson, A poor man's derivation of scaling laws for the Kondo problem, J. Phys. C 3, 2436 (1970).

[32] N. E. Bickers, Review of techniques in the large-N expansion for dilute magnetic alloys, Rev. Mod. Phys. 59, 845 (1987).

[33] K. G. Wilson, The renormalization group: Critical phenomena and the Kondo problem, Rev. Mod. Phys. 47, 773 (1975).

[34] R. Bulla, T. A. Costi, and T. Pruschke, Numerical renormalization group method for quantum impurity systems, Rev. Mod. Phys. 80, 395 (2008).

[35] A. K. Mitchell and L. Fritz, Kondo effect with a diverging hybridization: Possible realization in graphene with vacancies, Phys. Rev. B 88, 075104 (2013).

[36] A. A. Aligia, P. Roura-Bas, and S. Florens, Impact of capacitance and tunneling asymmetries on Coulomb blockade edges and Kondo peaks in non-equilibrium transport through molecular quantum dots, Phys. Rev. B 92, 035404 (2015).

[37] J. Fernández, F. Lisandrini, P. Roura-Bas, C. Gazza, and A. A. Aligia, Width of the charge-transfer peak in the $S U(N)$ impurity Anderson model and its relevance to nonequilibrium transport dot, Phys. Rev. B 97, 045144 (2018).

[38] L. Vaugier, A. A. Aligia, and A. M. Lobos, Spectral density of an interacting dot coupled indirectly to conducting leads, Phys. Rev. B 76, 165112 (2007).

[39] D. C. Langreth, Friedel sum rule for Anderson's model of localized impurity states, Phys. Rev. 150, 516 (1966).

[40] N. S. Wingreen and Y. Meir, Anderson model out of equilibrium: Noncrossing-approximation approach to transport through a quantum dot, Phys. Rev. B 49, 11040 (1994).

[41] D. Pérez Daroca, P. Roura-Bas, and A. A. Aligia, Relation between width of zero-bias anomaly and Kondo temperature in transport measurements through correlated quantum dots: Effect of asymmetric coupling to the leads, Phys. Rev. B 98, 245406 (2018). 